\title{
La "terca" pertinencia de la Pedagogía del Oprimido
}

\author{
Gustavo E. Fischman ${ }^{a}$ \\ Rebut: 12/05/2010 Acceptat: 17/05/2010
}

\section{Resumen}

Tomando como evidencia la cantidad y continuidad de las publicaciones sobre y acerca de Paulo Freire es fácil de constatar su persistencia y relevancia nivel mundial. En momentos donde los libros sobre pedagogía son olvidados casi al mismo tiempo en que son impresos y considerando que los trabajos más importantes de la obra Freireana fueron publicados en las décadas de 1960 y 1970, y el sinnúmero de controversias que acompañaron esos trabajos, la persistencia de un trabajo como Pedagogía del Oprimido, es de alguna manera "extraña". A partir de reflexiones de estudiantes de pedagogía este artículo presenta seis razones por las cuales Pedagogía del Oprimido, la prinicipal obra pedagógica de Freire y sus ideas siguen tercamente vigentes.

Palabras clave: Paulo Freire, Pedagogía Crítica, Formación Docente.

\section{The "stubborn" appropriateness of the Pedagogy of the Oppressed}

\begin{abstract}
The amount and continuity of publications on and about Paulo Freire provides evidence about his persistence and global relevance. At times where books on pedagogy are forgotten almost as soon as they are printed and considering that Freire's most important works were published in the decades 1960 and 1970, and the myriad of disputes that accompanied that work, the persistence of a book such as Pedagogy Oppressed, is somehow "estrange." Based on reflections of students in education, this article presents six reasons for why Pedagogy of the Oppressed, the most celebrated book by Freire and his ideas remain stubbornly in place.
\end{abstract}

Key words: Paulo Freire, Critical Pedagogy, Teacher Training.

\footnotetext{
${ }^{\text {a }}$ Arizona State University - EE.UU.
} 


\section{Introducción}

No hace falta decir que pocos en el ámbito comercializado de la academia contemporánea podrían pretender tener la eficacia pedagógica, espiritual y política que conserva el maestro e intelectual brasileño, Paulo Freire. Una década después de su muerte, todos sus libros siguen editándose (en más de 60 idiomas) y algunos se encuentran entre los títulos más vendidos para educadores. Una simple búsqueda en Internet nos ofrece muchas más referencias a Freire que a cualquier otro autor en el ámbito de la educación (por ejemplo, la entrada "Paulo Freire" da como resultado 1.800.000 páginas y "John Dewey" 1.600.000), y su nombre identifica a escuelas públicas y privadas, centros de investigación, organizaciones no gubernamentales y centros de educación inicial en más de 45 países.

Esos datos dan lugar a pocas dudas acerca de la popularidad y alcance, pero pareciera que el valor pedagógico del legado Freireano es menos aceptado. Celebrado y atacado con igual fervor por sectores de derecha y de izquierda, por educadores religiosos y laicos, intelectuales, marxistas, feministas, posmodernos, y hasta por algunos dentro de la corriente de la Pedagogía Crítica ${ }^{1}$. Freire ha sido citado y mal citado innumerable cantidad de veces. Sin embargo, me arriesgo a afirmar que las ideas Freireanas mantienen un atractivo tan singular, que casi seguramente en algún lugar y en este mismo momento un educador, en el aula, en el patio o en una clase universitaria diga con orgullo que está realizando un proyecto inspirado en la lectura de Pedagogía del Oprimido, sin dudas el libro más reconocido de Paulo Freire.

Desde su publicación, Pedagogía del Oprimido (PO) ha generado un debate vigoroso, no sólo sobre el contenido y la orientación de la obra de Freire, sino también sobre cómo entender sus ideas ${ }^{2}$. Joe Kincheloe, entre varios autores (Glass, 2001; McCowan, 2006), sostiene que:

Siempre ha habido un proceso de domesticación en el uso de PO, un proceso que se expresa en los esfuerzos antagónicos de "leer" Freire para alcanzar los objetivos y, el currículo de la pedagogía crítica. En un extremo profesores intentan despolitizar la obra de Freire de manera que resulta simplemente una amalgama de proyectos para el aula dirigidos por alumnos. En el otro extremo del continuum, algunos profesores han puesto de relieve las dimensiones políticas de Freire, pero ignorando el riguroso trabajo académico que propuso. Estos últimos esfuerzos se han traducido en un activismo social carente de sofisticación analítica y teórica. El trabajo académico que cultiva el intelecto y demanda análisis sofisticados se considera irrelevante en estas articulaciones anti-intelectuales de las ideas de Freire. Con estos problemas en mente la lucha para poner en práctica una pedagogía crítica freireana no debería buscar ninguna forma de "pureza" en la intención del autor. De hecho, Paulo Freire insistió en que lo criticásemos como un modo de mejorar sus ideas. Hacer honor a muchos de sus principios pedagógicos, sin santificarlo ni canonizar su obra es estar conceptualmente en la cuerda floja.

(http://criticalpedagogyproject.mcgill.ca/drupal-5.1/?q=node/37)

¿Qué diferencia a Freire de tantos otros intelectuales y educadores/as? ¿Cómo explicar tal persistencia, influencia y, sobre todo, tales esfuerzos de "domesticación"? 
¿Por qué su obra sigue influyendo, no sólo en las prácticas de quienes trabajan con poblaciones marginadas, sino también en los esfuerzos teóricos de muchos y muchas investigadoras? Tal vez la respuesta más simple es que si todavía estamos discutiendo sus ideas, es porque han sido producidas por un hombre "singular" que pertenecía a ese grupo especial de intelectuales que con palabras y acciones confieren significado y orientación a la sociedad. Estoy seguro de que Freire fue un educador y pensador excepcional, y sin duda fue uno de los mejores modelos de un intelectual comprometido (Fischman y McLaren, 2005) que uno puede encontrar, pero esto aún no explica qué características le confieren su "singularidad." ${ }^{3}$ En este texto voy a presentar y discutir algunas de las "razones" elaboradas en conjunto con un grupo de alumnos, que participan en el proceso de reflexión sobre la utilidad y la conveniencia de estudiar Pedagogía del Oprimido y otros textos de Paulo Freire en un programa de postgrado en los EE.UU ${ }^{4}$.

Lo que sigue son varias razones propuestas por los estudiantes como respuesta a la pregunta: ¿Por qué crees que es la Pedagogía del oprimido Freire (PO) sigue perdurando en el tiempo y generando debates? Voy a presentar cada una de las razones con las palabras proporcionadas por alguno de los/las estudiantes y, a continuación mis reflexiones en relación a las sugerencias de los estudiantes.

\section{Razón 1: Entender ideas ambiciosas}

"Siempre estamos leyendo libros y artículos repletos de grandes palabras, o sobre grandes problemas, pero en Pedagogía del Oprimido, Freire presenta una IDEA muy, muy, muy grande." (Claudia, 26 años chicana, terminando su maestría en Fundamentos de la Educación).

Varios participantes del seminario argumentaron que Freire los desafiaba con su mensaje simple pero que contiene una idea con enorme significado y consecuencias: "La educación funciona o bien como un instrumento que se utiliza para facilitar la opresión de las generaciones... o se convierte en 'una práctica de la libertad'" (Freire, 1993, p. 15).

La ambición epistemológica de PO -su compromiso intelectual para comprender y explicar todos los aspectos relacionados con los procesos educativos- atrae y desafía miradas optimistas y pesimistas, convocando a quienes están dentro y fuera de las aulas para, al mismo tiempo, criticar lo que existe para poder re-inventar modelos, prácticas, e instituciones educativas comprometidas con una idea de un futuro justo y posible. En el universo pedagógico de Freire, las ideas no son sólo abstracciones que nos permiten analizar la realidad, sino que son herramientas conceptuales concretas que producen una sensación de control y empoderamiento, cualidades que por cierto son escasas en muchas escuelas y las universidades contemporáneas.

Como educador, creo que este sentido de empoderamiento se relacionas con la ineludible dimensión ética en Freire. Si generaciones de maestras y educadores estuvieron y están dispuestas a comprometerse con el proyecto de PO, no es porque 
sufran de una contagiosa epidemia de confusión ideológica, o sean víctimas inocentes de las promesas vanas de un relato seductor de cambio educativo y redención. En mi lectura, la vigencia de PO no es fruto de confusión o seducción ideológica. Al contrario, educadoras y educadores continúan usando PO porque se identifican y hacen suyo el mensaje ético de Freire cuyas convicciones fundamentales afirmaban que el destino de nuestro mundo está vinculado a la condición de los oprimidos.

\section{Razón 2: Entender la complejidad pedagógica}

"Freire se entiende... incluso cuando uno no entiende todas las palabras en el libro. Leer a Freire me hace sentir algo sobre el poder de la enseñanza" (Rosa, 37 años, Mexicana estudiante de doctorado)

La capacitación y la sensación de control que ya habíamos identificado previamente regresa aquí en palabras de Rosa y parece relacionarse con el hecho de que aunque PO fue escrito en un lenguaje complejo -denso, con referencias eruditas y políticas (difícil incluso para los especialistas)- las profesoras y educadores que leen Freire sienten a menudo que entienden las ideas principales y que estas ideas pueden ser llevadas directamente a la práctica ${ }^{5}$. PO siempre ha sido percibido como un texto híbrido $^{6}$ y heterodoxo. Por otra parte, en un claro desafío a modelos pedagógicos ortodoxos y positivistas (incluidos marxistas y los así llamados modelos críticos y populares), las ideas pedagógicas y políticas de Freire resisten la reducción a modelos o métodos de fácil aplicación en cualquier contexto. Como señala acertadamente Adriana Puiggrós, "Los sujetos de la pedagogía de Freire no están reducidos a una existencia esencialista o a categorías inmutables: son sujetos complejos, determinados por múltiples factores, y que tienen diversas lenguas e historias" (1999, p. 123).

Freire utiliza un lenguaje teóricamente complejo y poéticamente atractivo y lo presenta un tanto paradójicamente, en términos sencillos. Freire sostenía que ser conceptualmente "simple", no implicaba el abandono de la coherencia en el uso de categorías teóricas, sino que permitía evitar posiciones reduccionistas. Sostenía que el lenguaje mediante el cual se presentan demandas y reivindicaciones, se proponen cambiar o se mantiene el orden del mundo, es un vocabulario siempre lleno de intenciones explícitas y con múltiples posibilidades de interpretación. Sin embargo, en vez de lamentar la multiplicidad de lecturas que provocó su obra, dio la bienvenida a la heterodoxia, a lecturas carnavalescas, y a la apropiación del lector de sus palabras. En cierto modo, la preocupación de Freire con la comunicación no se limitó a sus escritos, o incluso a cómo era leído, sino que abarcaba el reconocimiento de su influencia: "En el fondo, este debe ser el verdadero sueño todos los autores -ser leído, discutido, criticado, mejorado y reinventado por sus lectores" (1998: 31). 


\section{Razón 3: Pronunciar palabras artesanas}

"Freire fue un erudito, un profesor, pero también un artista, un artesano o tal vez ... como mi abuela que haciendo su artesanía, era capaz de contar historias que interpretaban el mundo, utilizando de forma selectiva (y esta es la palabra clave) lo que hubiera disponible, Freire hacía artesanía con las ideas."(Brian, de 35 años de edad, afro-americano y futuro psicólogo escolar).

La imagen de Brian de la artesanía refleja la "hibridez" analizada periódicamente por los estudiantes a lo largo de los años. En este caso, el carácter híbrido del proyecto PO refiere al modelo intelectual desarrollado por Freire que desplaza (por ósmosis) al viejo deweyano, pragmático y espiritualista, y más importante aún, desplaza los utópicos sueños modernizadores sobre educación de marxistas y cristianos. El lenguaje marxista o cristiano, o mejor; el lenguaje enraizado en categorías marxistas y cristianas, dio coherencia implícita y forma a $\mathrm{PO}$, proporcionándole la "estructura" emocionalmente profunda de gran parte de la política progresista que hay en el libro de Freire. PO fue además, un capítulo en la gran narración progresista y redentora de las escuelas de nuestro tiempo. En palabras de Freire:

"En última instancia, tengo que decir que tanto mi posición de cristiano como mi aproximación a Marx, no estaban en un nivel intelectual, sino en el nivel concreto. Yo no me acerqué a los oprimidos a causa de Marx, lo hice a causa de los oprimidos. Mi encuentro con los oprimidos me llevó hacia Marx, y no al revés" (1979: 74 y 75).

La hibridez artesanal que señalan Brian y algunos de sus colegas, también reconoce el hecho de que al igual que otros relatos clásicos sobre educación, PO tanto fomenta como antagoniza, el informe racional y optimista de la sociedad moderna y sus posibilidades. Lo propio de Freire -la afirmación de que una buena educación será un ejercicio en libertad a través de la praxis de los oprimidos en la que los educadores serían actores clave en el proceso de transformación educativa-, puede parecer difícil de creer para aquellos que siempre están denunciando la crisis de la educación y el estado desastroso de escuelas y profesores, pero sigue generando mucho más entusiasmo entre educadores y militantes que cualquier otro modelo pedagógico. Freire sigue resonando para los educadores y la PO parece posible, realizable, real -porque educadores y militantes pueden ver la forma en que la teoría informa a la práctica y viceversa.

\section{Razón 4: Pertenecer a un grupo con ideales similares}

"PO es como una insignia, una bandera, algo que me ayudó a establecer una conexión con otras personas, independientemente de cuál fuese su origen, cuál su raza, etnia, clase, orientación sexual, incluso si te gusta el kimchi o los tacos. Si ves alguien leyendo Freire, sabrás que ambos pertenecen al mismo grupo, que están del mismo lado"(Kim, 27 años de edad, es de Corea y estudia Educación Inicial).

Dos características sobresalientes y distintivas de PO se destacan en la descripción que hace Kim, de la influencia de la PO. En primer lugar, el libro se identifica como una herramienta intelectual para el desarrollo de "pedagogías de la resistencia." Pero 
la resistencia de Freire va más allá de negarse a obedecer las reglas de clase, porque, como él mismo escribió: "Es necesario entonces que nosotros tengamos la clase de resistencia que nos mantiene vivos. También es necesario que conozcamos cómo resistir de modo de seguir vivos, que nuestra comprensión del futuro no es estática sino dinámica, y que estamos convencidos de que nuestra vocación de grandeza y no de mediocridad es una expresión esencial del proceso de humanización en el que estamos insertos"(1999, p. 74)

En segundo lugar, la lectura y discusión de PO ha funcionado como una especie de "insignia" simbólica, que indica la voluntad de los educadores de comprometerse con las ideas del movimiento amplio de "educación popular/pedagogía crítica". Como un movimiento popular de base (especialmente en América Latina) el movimiento de educación popular/pedagogía crítica se identifica con Freire, pero una identificación tan fuerte no ha impedido que la generación de una miríada de variaciones desarrolladas en el diálogo con los profesionales, académicos y estudiosos. La educación popular de Freire se ha aplicado con todo tipo de grupos (niños, ancianos, mujeres, migrantes, indígenas, encarcelados) y entornos (rurales, urbanas, pobres, escuelas, universidades, cárceles, hospitales, etc.) Así, se ha fomentado un repertorio enorme de estilos, prácticas y estrategias. No obstante, mantiene una orientación pedagógica directivista no autoritaria con el objetivo de elevar la concientización como condición previa de la liberación.

Con el tiempo, la educación popular ha sido adoptada e incorporada en las iniciativas estatales, las políticas públicas (especialmente en el área de educación de adultos), y un sinnúmero de prácticas educativas sindicales, con poblaciones indígenas, organizaciones no gubernamentales, organizaciones comunitarias, grupos feministas, universidades y escuelas. Podemos nombrar algunos casos de experimentación pedagógica, por ejemplo, el Movimento Sem Terra en Brasil (Movimiento de los Sin Tierra), la iniciativa zapatista de educación popular en Chiapas (México), la iniciativa Cidadã escola en Porto Alegre (Brasil), la Universidad Popular Madres de Plaza de Mayo (Argentina), y la Universidad indígena Tupak Katari (Bolivia) que son buenos ejemplos de educación popular /crítica de inspiración freireana ${ }^{7}$.

Antes de presentar la razón siguiente, me gustaría retomar el debate sobre la "domesticación de Freire". Innumerables educadores populares, docentes de aula y profesores universitarios reclaman reinventar a Freire, y sin duda algunos de ellos lo hacen desde perspectivas comúnmente identificadas como de "sentido común" o "domesticadas". Etiquetar un enfoque como de "sentido común" o "domesticado" por lo general supone que los objetivos políticos, la noción de transformación, o la praxis han sido diluídas, lo que reduce $\mathrm{PO}$ a actividades grupales o prácticas paternalistas ${ }^{8}$. Identificando estos problemas, es importante reconocer que casi todos los esfuerzos por Freire-zar la educación- enfrenta las siguientes dos posibilidades: a) la multiplicación de voces que dicen estar haciendo "Freire" aumenta las oportunidades de que esos educadores reconozcan el papel de lo crítico y los retos para el desarrollo educativo, y b) no se puede "superar" el sentido común sin atravesarlo (Freire, 1997). 


\section{Razón 5: Experimentar tener poder}

No puede explicar completamente la experiencia de tener que lidiar con el tema educativo en un contexto real, como la India, enseñando en una colonia con mujeres.... Donde los ideales teóricos se unían a la realidad y yo podía ser lo que yo sabía que quería ser. Probablemente no sepa explicarlo bien, pero sólo sé que fue, en mi carrera, un verdadero punto de inflexión. Las ideas de Freire acerca de los maestros y los estudiantes, el aprendizaje y el poder de desarrollar modelos educativos impulsados por el conocimiento de los que tienen menos "poder" (pero un montón de conocimientos) resultó liberador para todos. (Elizabeth, estudiante blanca, madre de dos hijos y con amplia experiencia como investigadora con metodología cualitativa)

El reclamo insistente de PO sobre la naturaleza política de la educación no es una abstracción, o un "significante vacío" que pueda ser llenado por cualquier tipo de orientación. El ejercicio de las formas democráticas radicales de la educación depende tanto del rechazo de prácticas autoritarias como de las prácticas de laissez-faire. Freire (y muchos profesores) ven tales perspectivas duales como expresión de falsas opciones:

"Sólo porque yo rechazo el autoritarismo no significa que pueda caer necesariamente en la falta de disciplina; ni que rechace la anarquía implica que me dedique al autoritarismo. Como afirmé una vez: Uno no es el opuesto del otro. Lo contrario de cualquier autoritarismo manipulador o de la permisividad sin ley, es el radicalismo democrático"(1998: 64).

La sensación de poder observada por Elizabeth también se relaciona con el hecho de que la $\mathrm{PO}$ es rotundamente elocuente en su totalidad, pero particularmente en su oposición a la camisa de fuerza ideológica que supone pensar la enseñanza y el aprendizaje como emocionalmente neutros: "Es imposible enseñar sin el coraje de $\operatorname{amar}^{\prime \prime}(1998$ : 4). El amor de Freire, sin embargo, no debe entenderse como una forma de mimo paternalista que lleva a la indulgencia y a la comodidad, sino uno basado en una perspectiva radical de la relación entre educadores y educandos. Según Freire, el maestro es a la vez un estudiante, y el estudiante; un profesor. La naturaleza de sus conocimientos puede ser diferente, pero siempre que la educación sea un acto de conocimiento y no sólo la transmisión de hechos, los estudiantes y maestros comparten una situación similar en tanto productores y se vinculan entre sí a través de un diálogo pedagógico caracterizado por relaciones horizontales y dialógicas.

\section{Razón 6: Tener esperanzas realistas}

Lo que es tan hermoso de la mirada de Freire es que siempre nos recuerda que aunque las circunstancias sean terribles, podrían ser peores, pero también se pueden mejorar. En la Universidad vemos esperanza y espacio para la transformación. 
Conocemos nuestra historia. La rebelión y la juventud de Bosnia y Herzegovina requieren justicia y verdad.

En las montañas de la verdad nunca subes en vano:

o bien llegas un poco más alto hoy,

o deberás entrenar tu potencia para

ser capaz de subir un poco más alto mañana.

- Friedrich Nietzsche

(Ivana, abandonó Bosnia hacia Estados Unidos cuando tenía 15 años. Con frecuencia recordó al grupo que mantenía su corazón y su espera cerca de su país.).

Ivana menciona en su documento que se identificaba con la siguiente declaración en Pedagogía de la libertad:

"Me gusta el ser humano, porque yo sé que mi paso por el mundo no está predeterminado, establecido. Que mi destino no es algo dado sino algo que necesita ser construido y por lo que debo asumir una responsabilidad. Me gusta ser humano, porque estoy involucrado con otros en hacer de la posibilidad, historia. No simplemente resignación fatalista." $(1999,54)$.

Desde la publicación de $\mathrm{PO}$, los discursos pedagógico/políticos de Freire proponen una "esperanza" que exige cambios para un futuro que ya está "en" el presente. El discurso de Freire, insiste en la esperanza de una educación que para merecer ser llamada "educación" deberá contribuir a la creación de sociedades más justas y mejores. Al establecer un marco teórico para el presente, PO exige que cambiemos los aspectos opresivos de la vida cotidiana en las escuelas y en la sociedad mediante la participación en las luchas comunes del "aquí y ahora." Una mejor educación no necesita esperar una situación mágica que dará lugar a la emergencia de una conciencia utópica entre los oprimidos en el momento en que las circunstancias objetivas ideales se hagan realidad. Por el contrario, la utopía de Freire es realizable, pero sólo si se lee la palabra y el mundo en un proceso de concientización en evolución constante, que emerge de las condiciones concretas de la lucha cotidiana en la sociedad capitalista (Freire 1993). En la visión utópica de Freire de las escuelas, la tarea principal no es liberar a los demás mediante la aplicación de recetas ya hechas, sino para desarrollar la solidaridad con otros a través de las luchas diarias que se producen en las aulas, las escuelas, y las calles.

PO les dice a los profesores que la búsqueda de experiencias vívidas, incluso breves, de escolarización democrática (en un aula o en un solo distrito escolar) valen la pena. Estas experiencias nos enseñan no sólo a esperar más de las escuelas, sino también que mejorar la educación requiere del vínculo entre la participación individual y comunitaria con los objetivos de igualdad y solidaridad. También es preciso proporcionar acceso al conocimiento social y científicamente relevante y mejorar el rendimiento individual y socio-educativo. Creo que las palabras de Anita, otra de mis alumnos de postgrado, reflejan estos complejos sentimientos bastante bien: 
"Lo que me parece que hace único a Freire de entre los autores que habitualmente leemos es que sorprende al lector, porque con él hay un lugar para sonrisas, así como para la ira, pero no en las formas en que normalmente se espera. Por ejemplo, recuerdo que estaba cautivada por una investigación sobre la naturaleza de lo Indio, el abuso del mito de "minoría modelo", y los ejemplos de activismo surasiáticoestadounidense. Me encontré llorando en las con últimas frases del libro: "Los taxistas nos muestran cómo los inmigrantes y sus hijos pueden ser radicales en el vientre de la bestia. La suya es una pedagogía de la esperanza"(203). No estoy segura de por qué lloraba, pero creo que Freire habría sonreído."

\section{Reflexiones finales}

Freire escribe Pedagogía del Oprimido para denunciar las múltiples defectos del sistema bancario de educación, así como la habitualmente proclamada y nunca lograda 'igualdad de oportunidades' de la tradición liberal. Vale la pena recordar qué identifica el sistema bancario según Freire:

"La educación se convierte así en un acto de depósito, en el que los estudiantes son los depositarios y el profesor el depositante. En lugar de comunicarse, docente emite comunicados y depósitos que los alumnos reciben pacientemente, memorizan y repiten (p. 72). Considerando que la educación bancaria anestesia e inhibe el poder creativo, la educación cuestionadora implica un descubrimiento constante de la realidad. El primero mantiene la inmersión de la conciencia; el segundo se esfuerza por el surgimiento de la conciencia y la intervención crítica en la realidad" (p.62).

Si PO y otros textos posteriores, continúan inspirando a profesores, educadores y administradores es en gran medida debido a que las deficiencias del sistema educativo bancario siguen siendo la norma, no la excepción, y porque incluso hoy en día hay más profesores dispuestos a comprometerse y afirmar que otra experiencia educativa -una que sea más democrática, abierta, tolerante y creativa- no sólo es posible, sino necesaria. El discurso político-pedagógico de Freire proporciona ideales y metas realizables que permiten a los maestros y estudiantes, escuelas y comunidades reflexionar sobre las situaciones, palabras, sentimientos, e instituciones para desarrollar conscientemente espacios educativos institucionalmente más justos e interesantes, como partes de una nueva, cívica y utópica visión de la vida democrática. Un compromiso renovado con la justicia y la equidad en la sociedad y en educación es un movimiento de bienvenida para el conocimiento socialmente relevante, para el respeto por las diferentes perspectivas sobre las ciencias y las artes, para promover escuelas donde no se castigue el desacuerdo, donde prosperen el amor y el deseo de conocer, y donde la pasión por radicalizar de la democracia y crear alternativas más justas sea necesaria. 


\section{Notas}

${ }^{1}$ En una entrevista con Moacir Gadotti, Paulo Freire recordó la acogida de PO, "Algunos marxistas - y también no marxistas - que pensaban en términos puramente mecanicistas, me acusaron en los años setenta de ser, en el peor de los casos un idealista kantiano y en el mejor, un neo-hegeliano, a causa de mis propuestas de concientización que desafiaban la idea de que la superestructura condicionaba la conciencia "

2 Es pertinente reflexionar sobre la controversia "en torno a Freire." Cómo es posible que ciertos autores pueden generen debates y preguntas de este tipo: ¿Era un marxista? ¿Humanista? ¿Conservador? ¿Fue Freire el autor de un método, una metodología, una teoría, una pedagogía, una filosofía, un programa o un sistema? Cuando se le preguntó con cuál de esas denominaciones se sentía más cómodo, dijo: "Ninguno de ellos""Yo no inventé un método, o una teoría o un programa, o un sistema, o de pedagogía, o una filosofía. Es la gente la que necesita ponerle nombre a las cosas. "(Torres, 1997, p 2)

${ }^{3}$ Holst (2006) señala: "Si no se entiende el desarrollo de una pedagogía como la de Freire como un proceso colectivo situado socio históricamente, no somos capaces de ver las múltiples facetas del proceso en acción y nos limitamos a alimentar la innecesaria y distorcionada mística que se desarrolló en torno a Freire "(p.265).

${ }^{4}$ Más de 60 estudiantes participaron en el seminario de posgrado Re-Pensando Paulo Freire y la Política de Educación: La combinación de la lengua de la crítica con el lenguaje de la posibilidad entre 2002-2007, y aunque se trata de un seminario electivo que no cumple con algún requisito específico, es siempre la clase más concurrida de todas las clases que enseño.

${ }^{5}$ En esta cualidad existe el riesgo de reducir la complejidad de las ideas de Freire sobre la praxis, la caja de herramientas metodológica, transformando los objetivos políticos de justicia social en meros instrumentos, volver las teorías densas y de múltiples lecturas en consignas sobre "dominar la economía política de la educación."

${ }^{6}$ Como argumenta Daniel Schugurensky en una revisión de las contribuciones de Freire: "En los escritos de Freire encontramos, por ejemplo, elementos de la mayéutica socrática, el existencialismo filosófico, la fenomenología, el hegelianismo, el marxismo, la educación progresista y la teología de la liberación. Junto a Marx y la Biblia, están Sartre y Husserl, Mounier y Buber, Fanon y Memmi, Mao y Guevara, Althusser y Fromm, Hegel y Unamuno, Kosik y Furter, Chardin y Maritain, Marcuse y Cabral. A pesar de que Freire fue influenciado por estos y otros autores, su mérito fue combinar sus ideas en una formulación original "(1998, p. 23).

${ }^{7}$ No estoy afirmando que estas organizaciones sean ejemplos "puros" de prácticas Freirianas, sólo que no es posible aislarlos ni aislar su surgimiento de los modelos pedagógicos surgidos de las ideas de Freire y sus debates. 
${ }^{8}$ Leslie Barlett ofrece un ejemplo de esa situación:"Freire era un símbolo poderoso entre los educadores que conocí. Sus consignas tales como "enseñar insertos en la realidad de los estudiantes o enseñar a los estudiantes a leer 'la palabra y el mundo'" eran invocados con frecuencia durante las clases. En una ocasión, el premio en un concurso celebrado entre los alumnos del profesorado fue un breve folleto escrito por Freire. "Maestros" y "estudiantes" involucrados me dijeron que los programas de alfabetización de adultos en estas tres organizaciones estaban profundamente influenciados por las ideas de Freire. Es importante señalar, sin embargo, que los maestros de las ONG con los que he trabajado no tenían un conocimiento exhaustivo de la obra de Freire. Los profesionales solían estar familiarizados sólo con una pequeña parte de su obra Pedagogía del Oprimido. Pocos maestros, que no fuesen los de la universidad, habían leído más de un capítulo de alguno de los libros de Freire"(Barlett, 2005, 351)

\section{Referencias}

Bartlett, L. (2005) Dialogue, Knowledge, and Teacher-Student Relations: Freirean Pedagogy in Theory and Practice Comparative Education Review, vol. 49, no. 3. 344364

Fischman, G., \& McLaren, P. (2005). Rethinking critical pedagogy and the Gramscian and Freirean legacies: From organic to committed intellectuals or critical pedagogy, commitment, and praxis. Cultural Studies Bà Critical Methodologies, 5(4), pp. 1-22.

Freire, P. (1993). Pedagogy of the oppressed (New rev. 20th-Anniversary ed.). New York: Continuum.

-------- (1998). Pedagogy of hope: Reliving the pedagogy of the oppressed. New York: Continuum.

(1997). Pedagogy of the heart. New York: Continuum.

------- (1999). Pedagogy of freedom: Ethics, democracy, and civic courage.Lanham, MD: Rowman \& Littlefield.

Glass, R. D. (2001, March). On Paulo Freire's philosophy of praxis and the foundation of liberation education. Educational Researcher 30(2), 15-25.

Holst, J. D. (2006) "Paulo Freire in Chile, 1964-1969: Pedagogy of the Oppressed in Its Sociopolitical Economic Context" Harvard Educational Review, 76 (2) 243-270

McCowan, T. (2006) Approaching the political in citizenship education: The perspectives of Paulo Freire and Bernard Crick Educate Vol.6, No.1, 2006, pp. 57-70

Puiggrós, A. (1999) Neoliberalism and Education in the Americas, Boulder, Colorado, Westview Press 
Schugurensky, D. (1998) The Legacy of Paulo Freire: a critical review of his contributions. Convergence $31.1 \& 2: 26$.

Torres, R. M. (1997) The Million Paulo Freires. Convergence, "A Tribute to Paulo Freire", Vol. XXXI, N 1-2

\section{Nota Biográfica}

Gustavo E. Fischman, es profesor de Curriculum and Policy Studies en la Arizona State University. Sus campos de investigación engloban la educación comparada, estudios culturales, pedagogía crítica y el desarrollo de programas de investigación orientados a la participación y acción. Es autor de numerosos artículos y capítulos de libro sobre pedagogía crítica, temas de género en formación de profesorado y educación superior en revistas como Educational Researcher, Comparative Education Review, Journal of Education Policy, Gender and Education, Revista de Educacion, Cuadernos de Pedagogia, and Educaçao \& Realidade among others. Actualmente es miembro del Comité editorial y editor associado de las revistas: Education Policy Analysis Archives and Education Review. Es mimebro del comité internacional del Forum Mundial de Educación.

Correspondencia con el autor: Arizona State University.Mary Lou Fulton College of Education. Tempe Campus. PO Box 872011.Tempe, AZ 85287-2011; Phone: (480) 9655225. Fax: (480) 965-4942; Email: fischman@asu.edu 